\title{
Review of: "Dual perspective proteomics infectome profiling discovers Salmonella type III secretion system effector functions in macrophages"
}

\author{
Hai-Hua Ruan ${ }^{1}$ \\ 1 Tianjin University of Commerce
}

Potential competing interests: The author(s) declared that no potential competing interests exist.

This manuscript explored the temporal and pathogenicity island-specific expression in Salmonella and immune response proteins in macrophage from dual perspective of host and bacteria. Then, the fuction of SL1344_1263, SL1344_3112, SL1344_1563, and YnhG derived from bacteria and and Cd86, Cd40, Casp4, C3, IL-1 $\alpha$, and Cd69 derived from macrophage were depicted respectively. In total, this is a fairly good paper.

Major concern: (1) In proteomic sample preparation part in Materials and Methods, did the intracellular Salmonella be disrupted by probe sonication simultaneously with cell pellet preparation? If it is necessary to filter the whole intracellular Salmonella before precipitation? (2) Loss of test for identifying the whole intracellular bacteria were removed before preparation of cell pellet, because it is important to get rid of the disturb of bacteria.

(3) It will be really good to cite more new references. That is all my concerns. 